\title{
The Characterization of Steam Distillation as an Extraction Method to Extract Volatile Compounds from Prunella vulgaris and the Investigation of Their Anti-Tumorous Effect
}

\author{
William Chi Keung Mak, Sean Walsh \\ School of Life Science, University of Technology Sydney, Ultimo, New South Wales, Australia \\ Email: wckmak@gmail.com
}

How to cite this paper: Mak, W.C.K. and Walsh, S. (2021) The Characterization of Steam Distillation as an Extraction Method to Extract Volatile Compounds from Prunella vulgaris and the Investigation of Their Anti-Tumorous Effect. Journal of Biosciences and Medicines, 9, 120-142.

https://doi.org/10.4236/jbm.2021.98011

Received: June 15, 2021

Accepted: August 22, 2021

Published: August 25, 2021

Copyright $\odot 2021$ by author(s) and Scientific Research Publishing Inc. This work is licensed under the Creative Commons Attribution International License (CC BY 4.0).

http://creativecommons.org/licenses/by/4.0/

\begin{abstract}
Prunella vulgaris $(\mathrm{PV})$ is a perennial plant which is widely grown around the world. It has been widely used as a medicinal treatment for generations. Previous studies showed extracts from this plant had a wide range of therapeutic efficacy, including anti-tumorous effect. However, the volatile organic compounds (VOCs) extracted from it were rarely explored. This paper reports on the characterization of a steam distillation process to extract VOCs in PV and also the anti-tumorous effects of the PV distillate using the tetrazolium-based Cell Counting Kit-8 (CCK-8) as the test agent, when the VOCs were used to treat oral squamous cancer cells, SSC154. It was found that most abundant VOCs came out steadily and continuously for as long as the duration of the steam extraction could extend. However, some compounds such as benzaldehyde did show depletion as the distillation process progressed, while some compounds such as caryophyllene oxide was only sparsely found at the beginning of distillation. The PV distillate was mildly effective in its cytotoxicity to cancer cells SCC154, in a dosage dependent manner.
\end{abstract}

\section{Keywords}

Chinese Herbal Medicine, Steam Distillation, Prunella vulgaris, Xia Ku Cao, Volatile Organic Compounds, GC-MS, Cell Cytotoxicity, Cell Counting Kit-8

\section{Introduction}

Prunella vulgaris (PV) is a common herbaceous plant, which thrives in moist soil environments [1] [2] [3] [4]. The species vulgaris belongs to the genus Prunella, in the family Lamiaceae or Labiatae, which is commonly known as the 
mint family. Like many plants in the family, PV displays aromatic properties associated with its flowers, leaves and stems. The VOCs are responsible for the fragrant smell. It is Holarctic in distribution and is widely cultivated in Asia, extending as far north as Siberia and also south into the Indian sub-continent, and across to eastern Europe [5]. It additionally occurs in North America [6]. In China, the plant is harvested for medicinal purposes in April and May when the spica turns brown-red (and so the Chinese name Xia Ku Cao 夏枯草, which can be translated to "Wither in summer weed"). The spike consists of several whorls of calyx and bracts, each with flowers and seeds. The spikes, including the stems and seeds, comprise the "herb" component used in Chinese medicine. After harvest, unwanted impurities and dirt are removed, and the herb then dried in the sun before packaging.

Prunella vulgaris is a commonly used medicinal herb in traditional east Asian medicines. It belongs to the Clearing Heat and Purging Fire category in Chinese medicine terms [7] and consequently prescribed for signs and symptoms affecting the upper body, including eye pain and eye redness, headaches and dizziness [8] [9]. Its use extends into oncological settings as a concomitant for treatment of tumours, used in addition to standard care with western medical interventions; for examples, in transcatheter arterial chemoembolization [10], and in combination with chemotherapeutic agents [11].

Biomedical studies report the various clinical applications of PV. These include the antioxidative effect of phenols found in PV [12], antiviral effect against human immunodeficiency virus (HIV) [13] [14] [15], anti-inflammatory effect by induction of heme oxygenase [16], anti-tumorous effects [17], and immunomodulatory activities [18].

Research studies have also focused on the identification of chemical components in PV. Early studies by Kojima and Ogura [19] using methanol as the extraction solvent and then applying fractionation by chromatography, reported the presence of triterpenoids (such as sterols, ursane, and oleanane). More recent studies have reported also the presence of triterpenes [9] [20] [21] [22]. These included rosmarinic acid, maslinic acid, corosolic acid, betulinic acid, oleanolic acid and ursolic acid. Golembiovska et al. [5] investigated the chemical composition of PV grown in Ukraine using GC-MS, looking at each section of the plant (spikes, leaves, stems and root). The presence of squalene, myristic acid, spathulenol, viridifloral and germacrone were reportedly found in the various parts of the plant. In other studies, other bioactive compounds identified in PV have included polyphenols [12] [23] [24], polysaccharides [14] [25] [26] [27], and flavonoids [17] [28] [29].

VOCs produce distinctive fragrance and are found in other plants used in culinary and in traditional medicines and have been extensively studied. These include, as examples, Artemisia argyi [30], clove, cinnamon, nutmeg, basil, oregano, thyme [31]. Only a few studies have focused on PV's VOCs. Of the few identified, they have focused on the identification of VOC composition [5] [32] 
[33] [34]. Studies on the pharmacological effects of the volatile compounds of $\mathrm{PV}$ are also scarce [35], despite the importance of the herb in a range of herbal formulae used in traditional medicine systems still in common use throughout the world [7].

Distillation is a conventional method used to extract volatile compounds in laboratories. The traditional method to prepare herbal formula in China is, however, by decoction, which means boiling the herbs in water. An early record of the use of distillation as a method to prepare PV as a medicinal treatment is from the Qing Dynasty [36]. In the book, Zhao wrote, "Use the whole plant of PV and use distillation to get the fragrant liquid". The medicinal effects he described were, "healing scrofula, pain of eyes, avoidance of bright light", which is similar to the indications of the herb when prepared with contemporary decoction methods described in the literature [37].

No studies on steam distillation were identified in the literature. Distillation, as a whole, is not the conventional method to prepare Chinese herbal medicine; thus, this is reflected in the limited literature reporting this method [35] [38] [39] [40]. They concentrated on how the distillation methods effect compound extraction. However, they did not raise question on how might the use of different distillation methods effect compound extraction. This study tries to address this question.

\section{Objective of This Research}

The objective of this research is on the characterisation of the steam distillation process itself and the pharmacological anti-tumorous effect of the VOCs collectively present in the distillate. (That is, looking at the whole of the anti-tumorous effects of the distillate, reflecting traditional medicinal practices rather than focusing specifically on individual organic compounds). The particular focus on the anti-tumorous effect reflects the continued interest in cancer research.

\section{Materials and Methods}

Figure 1 shows the workflow of the procedures undertaken in this investigation for quick reference.

\subsection{Materials}

Dried inflorescence of Prunella vulgaris was obtained from TDT Australia Pty Ltd., Rydalmere, NSW, a reputable importer and supplier of herbal traditional medicines in Australia. The herb was grown in China and collectively batched from different sources. Preparation of the herb inflorescence for testing required the whole herb to be finely chopped using a standard chopper (Russell Hobbs Classic chopper RHMFP2).

The authentication of the herb was carried out by thin-plate chromatography following the procedure in the Pharmacopoeia of the People's Republic of China, p. 359, 2010 edition [41]. Rosmarinic acid purchased from Sigma-Aldrich 


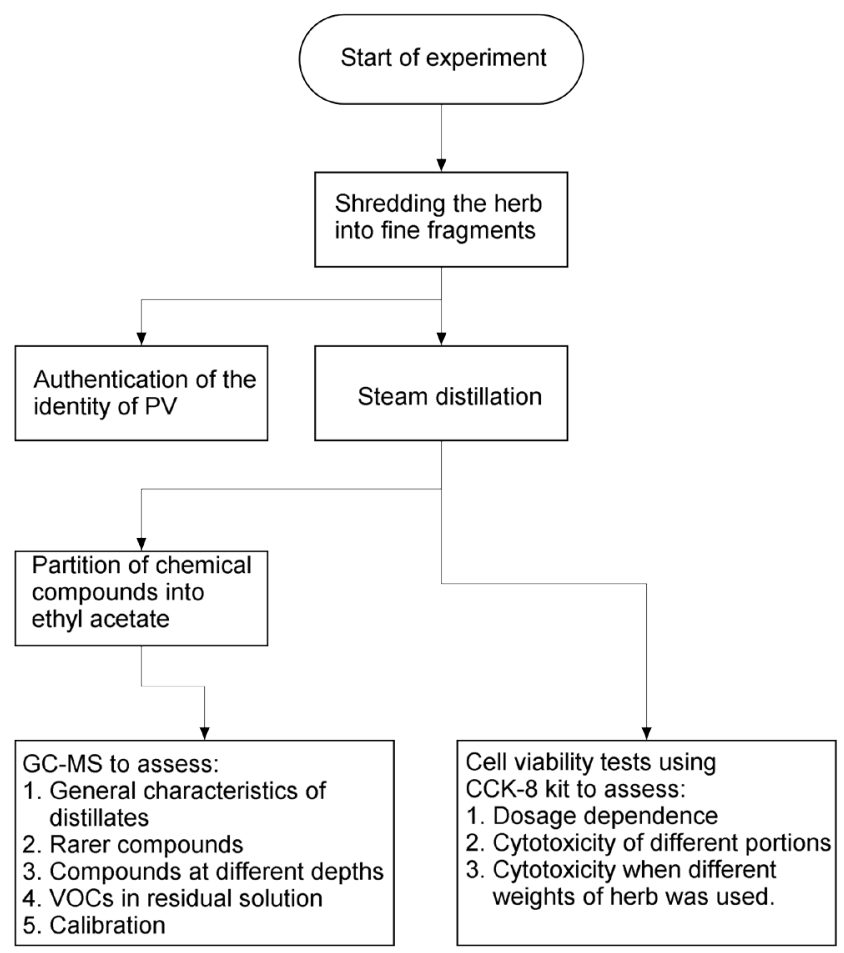

Figure 1. Flowchart showing the workflow of the procedures undertaken.

(R4033-10MG lot \# BCBV7877) was used as the reference standard in the procedure.

Gas chromatography-mass spectrometry was carried out using a GC-MS machine manufactured by Agilent ${ }^{\mathrm{TM}}$ Technologies (6390N Network GC System and 5973 Network Mass Selective Detector).

To characterize the cell cytotoxicity of the PV distillate on cancer cells, tetrazolium-based testing reagent, Cell Counting Kit-8, was purchased from Sigma-Aldrich. The cancer cell line used is the SCC154 line, obtained internally in the university.

\subsection{Methods}

\subsubsection{Identification of the Herb}

A standardised procedure described in the Chinese Pharmacopoiea (p. 359, 2010 edition), was followed [41]. Chemical components of $5 \mathrm{~g}$ of finely chopped PV were extracted by ultrasonification in $30 \mathrm{ml}$ of ethanol. The extract was dried and redissolved in ethanol as the test solution. The test solution was subjected to thin layer chromatography to separate out the components. Rosmarinic acid purchased from Sigma Aldrich was used as a reference standard. Comparison of the traces formed by the test solution and the reference solution confirmed the presence of rosmarinic acid in the herb.

\subsubsection{Extraction of Volatile Compounds by Steam Distillation}

As the Russel Hobbs chopper has only an ON/OFF switch on the machine, the 
chopper was run to shatter the spica for 5 minutes each time to keep the consistency of the fineness of the fragmented herb.

Dried shredded PV herb was exposed to steam to extract water soluble volatile compounds (refer to Figure 2 for the experimental setup). The setup involved a two-necked flask with de-ionized water being heated by an electric heater (with temperature setting adjusted to give a constant distillate collection rate of around $50 \mathrm{ml}$ every 50 minutes across different distillation runs). Attached to the flask was a two-ended reservoir flask containing the herb. Rising steam passed through different quantities of finely chopped PV. The steam was then collected in a secondary condenser branch cooled by running room temperature water from the tap. In order to characterise the continuous process of the distillation, the distillate was collected in successive $50 \mathrm{ml}$ portions, with distillation run identifier, weight of herb used and sequence number, recorded on the tubes. In most cases, a minimum of 9 distillate portions (of $50 \mathrm{ml}$ each) were collected, or $450 \mathrm{ml}$ of distillate in total in each run (see discussion in Section 3.3.1 below for exception).

Different herb quantities of $2 \mathrm{~g}, 5 \mathrm{~g}, 15 \mathrm{~g}, 25 \mathrm{~g}, 35 \mathrm{~g}$ were used in separate distillation runs. The use of different quantities enables the dosage effect to be determined. These quantities were chosen by successive analyses of data and assessment of results after individual distillation runs, as trends of results progressively emerged.

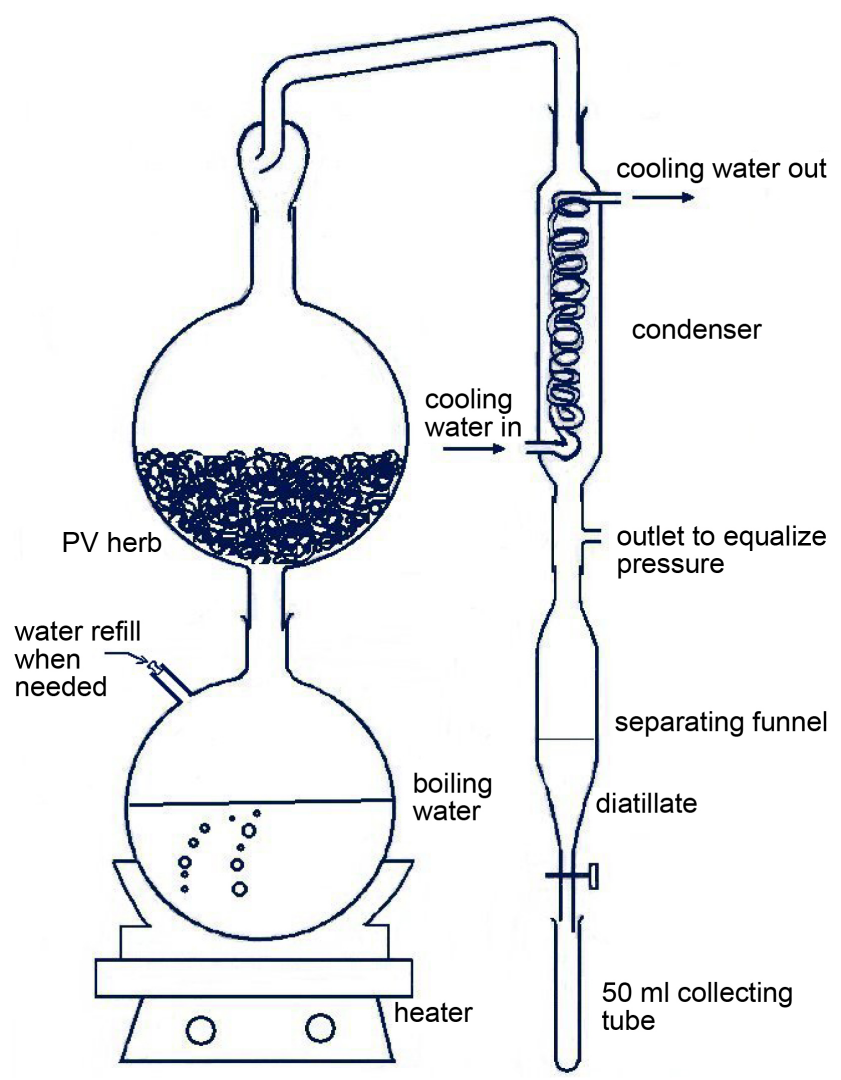

Figure 2. Apparatus setup of the steam distillation process. 


\subsubsection{Preparation of the GC-MS Test Solution}

Partition of the chemical compounds inside the aqueous distillate into ethyl acetate was done before the GC-MS analysis. $1 \mathrm{ml}$ of distillate from a chosen $50 \mathrm{ml}$ distillate portion, and $1 \mathrm{ml}$ of ethyl acetate were each pipetted into a test tube. The mixture was then vortexed for 1 minute to mix well and rested until two immiscible layers formed. The top ethyl acetate layer with the fractionated VOCs was then withdrawn by a pipette and delivered into a second test tube. Remaining water was removed by adding anhydrous potassium sulfate powder before the dehydrated solution was subjected to GC-MS analysis.

The GC-MS analysis was done as soon as possible after the distillate was obtained. However, when this was not practical (for example, during the waiting time while the GC-MS machine was occupied by another previous run), the distillate in its collection tube was stored in a refrigerator with the temperature set at $5^{\circ} \mathrm{C}$. This refrigeration attempted to avoid evaporation of VOCs or minimize any possible chemical reaction within the distillate while waiting. Otherwise, after partition into the ethyl acetate solvent, the samples (in ethyl acetate) were stored in a freezer with the temperature setting at $-21^{\circ} \mathrm{C}$ (for example, when the waiting time was much longer; say, overnight).

\subsubsection{GC-MS Analysis}

GC-MS analyses were performed with an Agilent ${ }^{\mathrm{TM}}$ gas chromatography machine in tandem with an Agilent mass spectrometer. The gas chromatography machine was equipped with a capillary column with dimensions of $30 \mathrm{~m} \times 0.25$ $\mathrm{mm} \times 0.25 \mu \mathrm{m}$. The settings were as follows: Split mode injection; helium was used as the carrier gas at a flow rate of $1.2 \mathrm{~mL} / \mathrm{min}$ under a pressure of $6.57 \mathrm{psi}$ and an average velocity of $31 \mathrm{~cm} / \mathrm{s}$. At the inlet, the heater setting was at $250^{\circ} \mathrm{C}$, at a flow rate of $27.7 \mathrm{~mL} / \mathrm{min}$ and a pressure of $4.24 \mathrm{psi}$. The temperature profile of the oven was: Temperature held at $50^{\circ} \mathrm{C}$ for $3 \mathrm{~min}$; then, ramping up to $250^{\circ} \mathrm{C}$ at a rate of $10^{\circ} \mathrm{C} / \mathrm{min}$; holding at $250^{\circ} \mathrm{C}$ for $3 \mathrm{~min}$; then, ramping up to $280^{\circ} \mathrm{C}$, where the temperature was at hold for a further $5 \mathrm{~min}$, thus, making the total oven time of 34 minutes.

The mass spectrometer was set at electron impact mode, with a scan range between $30-500 \mathrm{amu}$, with a data rate of $20 \mathrm{~Hz}$, and the detector set point at $280^{\circ} \mathrm{C}$. The temperatures at the MS source and quad, were at $230^{\circ} \mathrm{C}$ and $150^{\circ} \mathrm{C}$ respectively.

The volatile compounds were identified by comparing the mass spectra obtained from the GC-MS runs with the mass spectrum library of the National Institute of Standards and Technology (NIST08), available internally in the software supplied together with the machine.

\subsubsection{Cell Culture}

The oral squamous cancer cell line SCC154 was used to study the cytotoxic effect of the VOCs in the PV distillate. Given the traditional indications of PV to treating diseases of the upper body and head, it was decided to select a cancer cell line from this region. The cells were maintained in DMEM medium supple- 
mented with $10 \%(\mathrm{v} / \mathrm{v}) \mathrm{FBS}$ at $37^{\circ} \mathrm{C}$ in a humidified incubator with $5 \% \mathrm{CO}_{2}$.

\subsubsection{Calibration Using an Internal Standard}

Many abundant compounds present in the distillate can be chosen as internal standard for calibration of any further distillates obtained. Dodecane was chosen as the internal standard, as it appeared in all samples and was identified in their corresponding chromatograms. A known amount in weight of the standard compound, dodecane, was measured (so that the concentration of the final standard calibration concentration can be calculated), dissolved in a known amount of and diluted by ethyl acetate. GC-MS analysis was then done to obtain the abundance corresponding to this known concentration of dodecane. Then, the dodecane solution was then repeatedly diluted and analyzed by GC-MS, to obtain a calibration standard curve (Figure 3 ). In doing the biological tests described below, the distillate used to treat the cells was first calibrated using this calibration curve.

\subsubsection{Determination of Cell Viability}

The test reagent used to do the task was the Cell Counting Kit-8, (product id: 96992), a product purchased from Sigma-Aldrich Inc., Australia. It allows for very convenient colorimetric assays by utilizing the highly water-soluble tetrazolium salt WST-8, which is converted to a formazan dye, which is soluble in tissue culture medium, due to reduction in the presence of an electron carrier in the process of metabolism of cells. The absorbance at $450 \mathrm{~nm}$ is proportional to the number of viable cells in the culture medium.

\section{Calibration standard curve}

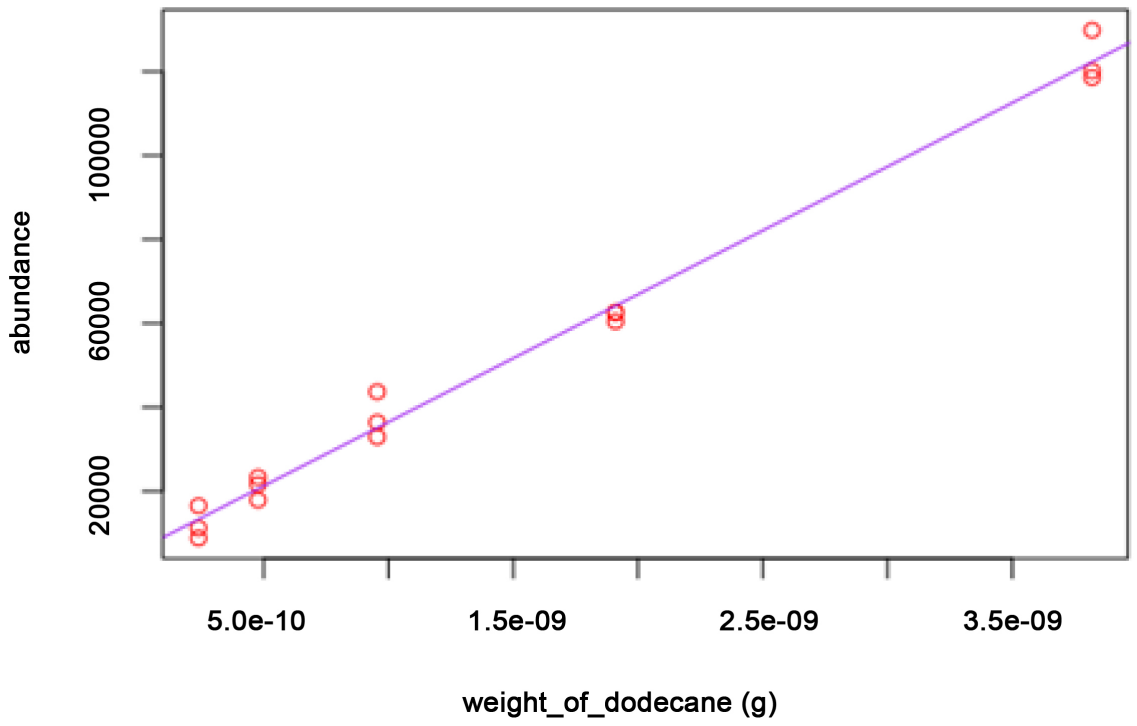

Figure 3. The calibration standard curve. The formula is: $a=c_{1}{ }^{\star} g+c_{0}$, where a $=$ abundance in the GC-MS given arbitrary units. $g=$ weight of dodecane in $5 \mu \mathrm{L}$ of injection distillate fluid. $c_{1}=$ slope of interpolation curve, 3.04345e $+13 . c_{0}=y$-intercept, $6.036528 \mathrm{e}+3$. Variance $\sigma^{2}=2.25365097 \mathrm{e}+8$. 
The assay used followed the procedures in the leaflet "Product Information" which comes with the purchased product. Briefly speaking, $100 \mu \mathrm{l}$ of cell suspension at a concentration of 5000 cells/well were dispensed into the wells of a 96-well tissue culture plate; three in a set so that data in triplicates could be obtained. The plate was pre-incubated in an incubator for 24 hours. The incubator had a fixed setting with an ambient temperature of $37^{\circ} \mathrm{C}$ and $5 \% \mathrm{CO}_{2}$. After incubation, $10 \mu \mathrm{l}$ of various concentrations of the PV distillate was added into the medium in the wells; also, three in a set. The concentrations of the PV distillate were the full-strength distillate directly from the distillation for all tests, except in the test for dosage dependence. In this case, 1, 0.5, 0.1, $0.01 \mathrm{v} / \mathrm{v}$ of the full-strength distillate diluted by DI water were used; so making 4 sets of 3 wells each. The plate was further incubated for 48 hours. CCK- 8 reagent taken from the refrigerator was put on a bench top at room temperature for half an hour to allow thawing. Thawed CCK-8 was added to each well and incubation was allowed for another 3 hours. Finally, measurements of the absorbance at $450 \mathrm{~nm}$ were completed.

\subsubsection{Statistical Analysis}

For the cell viability assays, the experiments were performed in triplicates. The statistical analysis was performed using one-way analysis of variance (ANOVA) test. The threshold significant p-value was chosen to be 0.05 .

\section{Results}

\subsection{Identification of the Herb}

The identification followed that described in the Chinese Pharmacopoiea ( $\mathrm{p}$. 359, 2010 edition) [41]. Thin-plate chromatography was used as a step to verify the identity of the herb. The experiment was repeated for a total of 4 times to compare the retention factors of the test solution with the reference solution of rosmarinic acid. The identity of the herb was thus verified.

\subsection{Calibration Using an Internal Standard}

A calibration standard curve was obtained as shown in Figure 3. The distillate used to treat the cells in the biological tests was first calibrated using this calibration curve.

As the abundance of dodecane in the distillate measured by GC-MS is around 20,000 (when $15 \mathrm{~g}$ of herb was used). Thus, it is equivalent to have a concentration of around $0.1 \mathrm{ng} / \mu \mathrm{L}$.

\subsection{Characteristics of the Distillate and the Residue}

\subsubsection{General Characteristics}

GC-MS analyses were done on alternate portions of distillate collected in the distillations. Experiments were repeated and data were taken in triplicate sets to identify and get the abundances of the different VOCs in the distillate. A typical chromatogram is shown in Figure 4. 


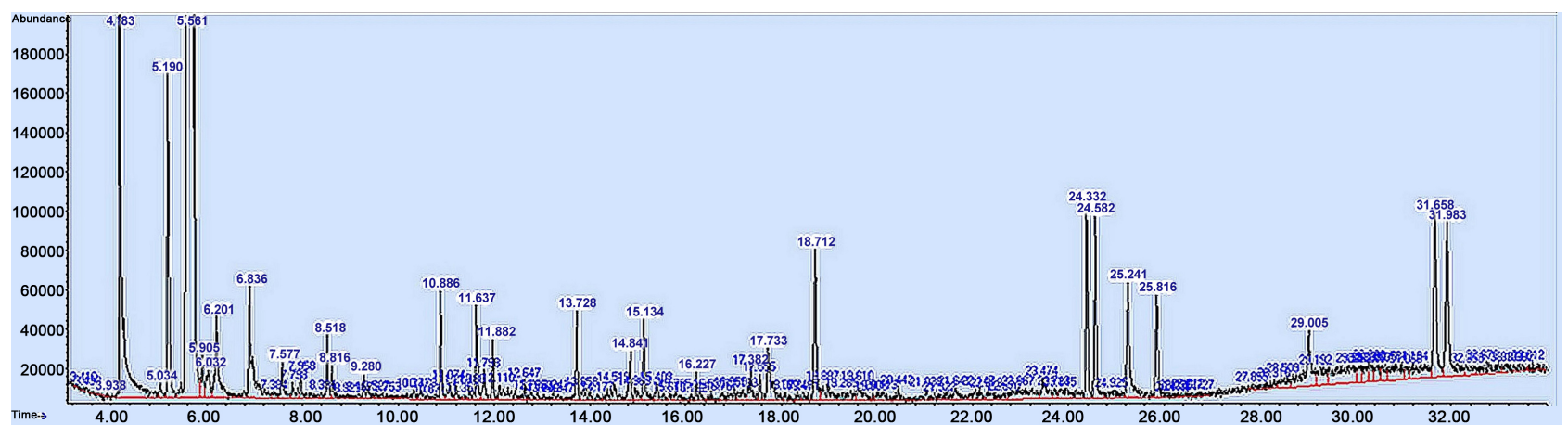

Figure 4. A typical chromatogram from a GC-MS run. The amount of herb used was $15 \mathrm{~g}$. The sample was taken from the fourth portion of a distillation run. The numbers shown at the peaks are the retention time. Retention time of some VOCs discussed in this article are: Furfural (3.46 $\mathrm{min})$, anisole $(6.04 \mathrm{~min})$, benzaldehyde $(6.85 \mathrm{~min})$, decane $(7.48 \mathrm{~min})$, eucalyptol (8.15 min), dodecane (10.8 min), tetradecane (13.84 min), caryophyllene oxide (16.18 min), hexadecane $(16.27 \mathrm{~min})$.

From the GC-MS analyses, there is a diverse variety of volatile compounds in Prunella vulgaris. Some of these compounds identified included alkanes such as decane and dodecane. These have been previously reported [32] [33]. Other rarer compounds identified included eucalyptol, anisole, furfural and benzaldehyde. This project focused on the pharmacological effect of the volatile compounds of $\mathrm{PV}$ as a whole, reflecting the clinical use of $\mathrm{PV}$ in Chinese medicine, which favours the idea of making use of the synergistic effects of different compounds in a single herb, or even the synergistic effects of many herbs together in herbal formulae [7]. Consequently, further extensive identification of other compounds was not pursued at this stage of the project. Additionally, previous studies have reported identified volatile compounds in PV [32] [33] [35] [38] [39] [40].

To show the efficiency of extracting the volatile compounds when different amounts of herb are used, distillations using $2 \mathrm{~g}, 5 \mathrm{~g}, 15 \mathrm{~g}, 25 \mathrm{~g}$, and $35 \mathrm{~g}$ of PV herb were done to characterize the dependence of the abundances of volatile compounds extracted on the amount of herb used. Figure 5 shows the abundances of four alkanes which were extracted. These 4 alkanes were chosen as their identities were confirmed by the comparison of the chromatograms with those obtained with the standard compounds which were available in the local university chemistry laboratory. It can be seen (Figure 5 \& Figure 6) that the abundances of the VOCs extracted did not change much as the distillation process proceeded. To verify this observation, distillation run was prolonged to collect up to 19 distillation portions for the case when $5 \mathrm{~g}$ of herb was used, to see if the amounts of VOCs extracted did diminish after a long distillation process.

Also, as observed for this experimental setup, there was an optimal amount of PV herb used which extracted the most abundant amounts of VOCs. This optimal amount was found to be about $15 \mathrm{~g}$. The use of more herb (25 g and $35 \mathrm{~g}$ ) resulted in a decrease in efficiency in the extraction; smaller abundances of the VOCs were collected. 


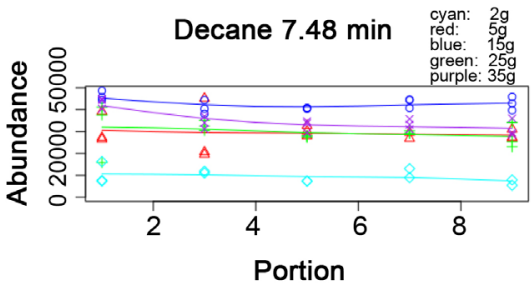

(a)

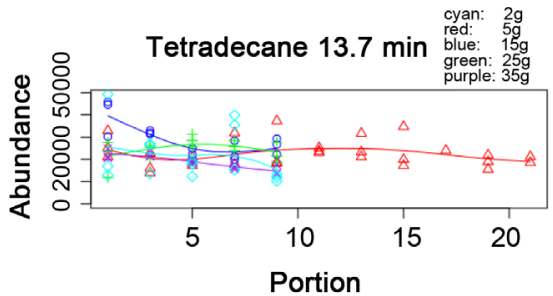

(c)

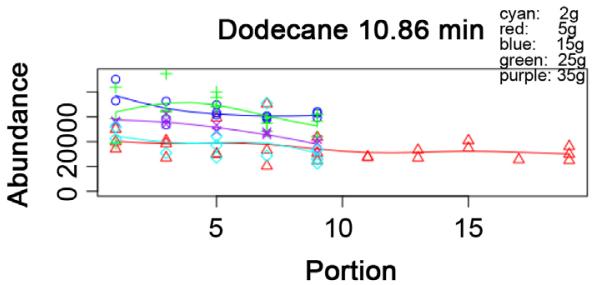

(b)

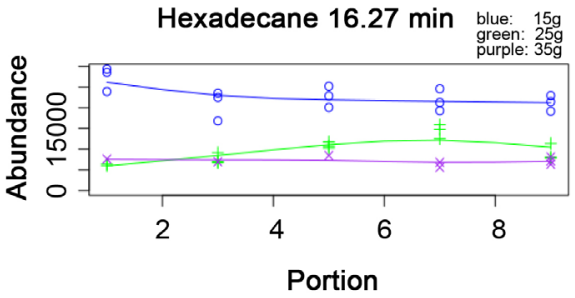

(d)

Figure 5. The abundances of 4 volatile alkanes: (a) decane, (b) dodecane, (c) tetradecane and (d) hexadecane obtained in successively collected $50-\mathrm{ml}$ portions during the distillation process. Different curves represent cases when different amounts of herb were used: $2 \mathrm{~g}, 5 \mathrm{~g}, 15 \mathrm{~g}, 25 \mathrm{~g}$ and $35 \mathrm{~g}$ respectively. The numbers appear in the sub-titles are the retention times of the compounds in minutes. The smoothing lines were fitted to the data using cubic smoothing splines with a degree of smoothing parameter equal to 0.4.

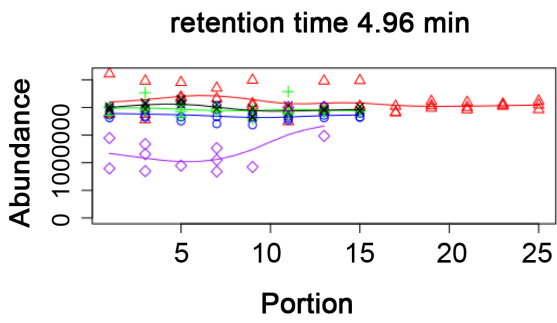

(a)

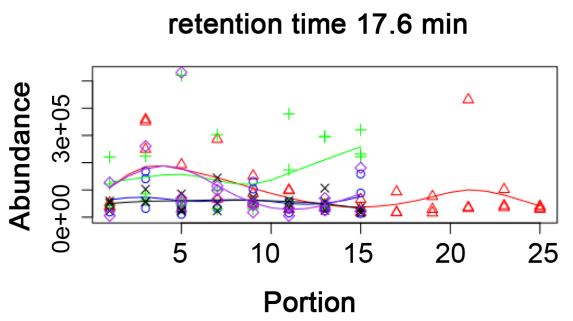

(c)

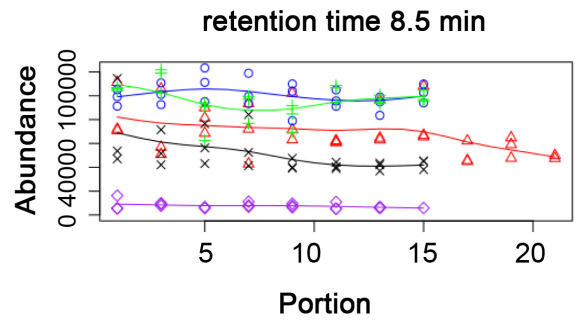

(b)

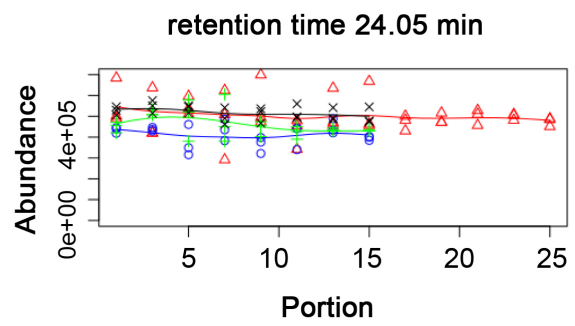

(d)

Figure 6. The abundances of 4 volatile compounds, the identities of which were not confirmed. So, only the retention times of the compounds are indicated. They were collected in successive $50-\mathrm{ml}$ portions during the distillation process. Different curves represent cases when different amounts of herb were used: $2 \mathrm{~g}$ (color code: cyan), $5 \mathrm{~g}$ (red), $15 \mathrm{~g}$ (blue), $25 \mathrm{~g}$ (green), $35 \mathrm{~g}$ (purple) and $45 \mathrm{~g}$ (black) respectively. The smoothing lines were fitted to the data using cubic smoothing splines with a degree of smoothing parameter equal to 0.4 .

Figure 6 shows abundances of 4 more compounds other than the 4 alkanes shown in Figure 5. The identities of these compounds were not confirmed by comparisons of their chromatograms with standard compounds. So, as these 
compounds are not properly identified, only their retention times in the GC-MS were shown. Comparison of Figure 6 with Figure 5 shows that there is no contradiction with the observations made above.

\subsubsection{Rarer Volatile Compounds}

The volatile compounds discussed above did not show much depletion in abundances as the distillation process proceeded. However, some rarer compounds in the PV herb, which showed obvious depletion as distillation continued, were noted. They include benzaldehyde, caryophyllene oxide, eucalyptol, anisole and furfural. The compound identities of them were confirmed by comparisons of the chromatograms with those of standard compounds, except eucalyptol, which was confirmed by the repeated reports by the analysis software of the GC-MS machine only. Figure 7 shows the abundances of benzaldehyde for portions obtained during the distillation process. It can be seen that the abundances of benzaldehyde did show obvious decline as the distillation process proceeded. Also, benzaldehyde was not found when the dosage of PV herb used was below $25 \mathrm{~g}$. This shows its relative scarcity.

Other volatile compounds identified appeared even less frequently. Consequently, their abundances have been represented differently from those noted above in Figures 5-7. Instead, in Figure 8, their abundances are shown as sunflower plots, in which the number of sunflower "petals" is directly proportional to the abundances. Different compounds are indicated by different colours in the plots. The "replicate" as indicated in Figure 8 refers to the repeat number in a set of triplicate data. These compounds were absent from PV samples of amounts under $25 \mathrm{~g}$. Caryophyllene oxide, anisole and eucalyptol were found only in the first portion at the beginning of the distillation process, except briefly in two instances in the second and the third portions (for caryophyllene oxide), and once in the third portion (for anisole), and briefly in one instance in the second portion (for eucalyptol). Anisole and furfural also appeared only after a large amount of PV herb (45 g) was used. Both mainly only appeared in the first

\section{Benzaldehyde 6.97 min}

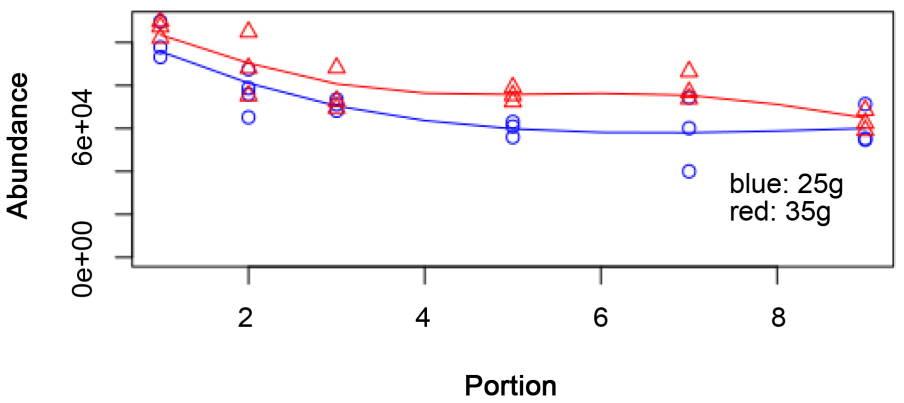

Figure 7. The abundances of benzaldehyde, obtained in successively collected $50-\mathrm{ml}$ portions during the distillation process. Only 2 curves are shown, as benzaldehyde was not found when the PV dosage used was below $25 \mathrm{~g}$. 
Abundances of rare compounds, $25 \mathrm{~g}$ of herb used

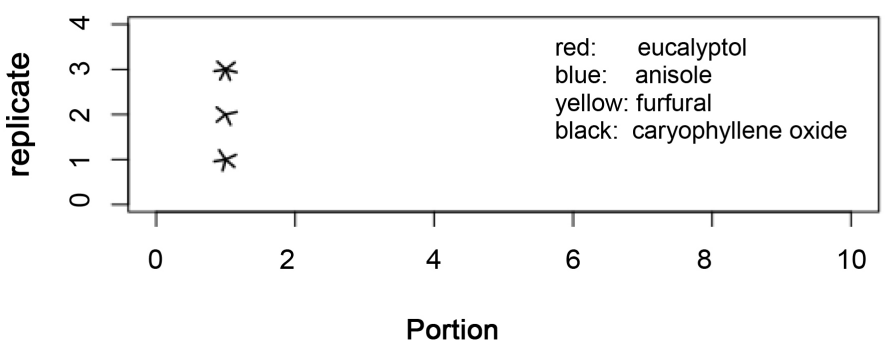

(a)

Abundances of rare compounds, $35 \mathrm{~g}$ of herb used

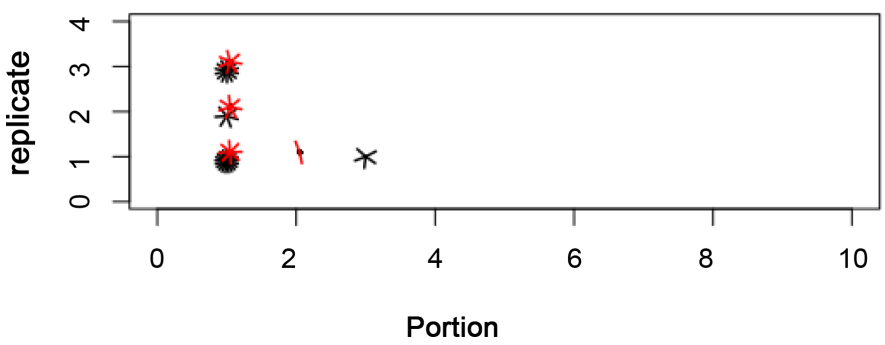

(b)

Abundances of rare compounds, $45 \mathrm{~g}$ of herb used

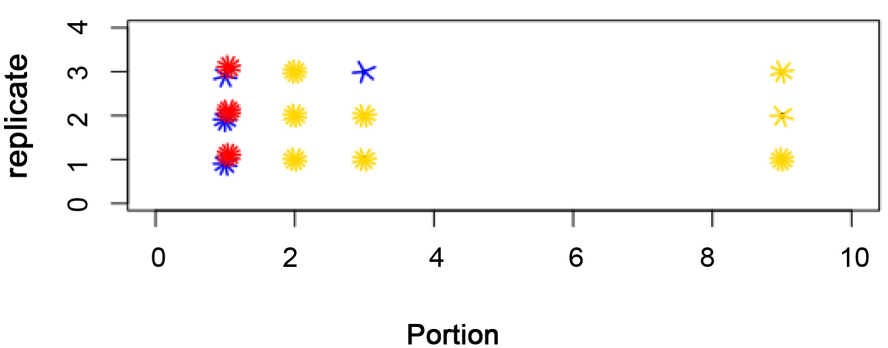

(c)

Figure 8. The abundances of rarer volatile compounds in PV herb, indicated as sunflower plots, as a function of the portions successively collected. The abundances are proportional to the number of "petals" at each sunflower (data point). The measurements were made in triplicates, which are indicated as the y-axis, with the label "replicate". The choice to show the data in this format is to highlight the fact that the compounds were not found in each of the triplicates even if they were sampled from the same portion.

3 portions. For furfural, it appeared also, however, briefly in the ninth portion (reason unknown).

\subsubsection{The Concentration of Volatile Compounds at Different Depths Inside the Distillate}

Triplicate samples were taken from the surface, the mid-level (as judged from the graduated marks on the containing tube) and the bottom of the test tube containing the ninth portion of the distillation using $15 \mathrm{~g}$ of PV herb. The samples in the tube should be allowed to settle well (allowed a waiting time of more 
than 5 minutes) before they were taken, and GC-MS analyses were done on these samples. Figure 9 shows the results. It is a plot of the abundance versus retention time for the three depth levels. Since the retention time relates directly with the molecular weights; thus, the plot indicates the abundances of volatile compounds of various molecular weights at the three different depth levels. It can be seen that for most volatile compounds, the concentrations of them were the highest at the bottom of the solution, which means that they sank to the bottom, indicating that they had densities higher than water. The exceptions were those compounds which were light (having small retention time). This means light compounds floated to the top.

\subsubsection{Volatile Compounds in the Residue Solution}

GC-MS analysis was also done on the residue solution left behind in the distillation process. It was found that volatile compounds found in the distillate were also found in the residue liquid, but with reduced variety and amounts. The reduction was more noticeable especially for the lighter compounds (with retention time less than 10 minutes). So, it was confirmed that not all the volatile compounds were carried over to the collecting side of the setup, but they dropped back into the residue liquid after they were extracted by the steam rising from below.

\subsection{Cell Viability Tests}

To characterize the anti-tumorous effect of the distillate of PV, the Cell Counting Kit-8 (CCK-8) was used to investigate the cytotoxicity of the PV distillate on cancer cells from the oral squamous cancer cell line SCC154.

\subsubsection{Dosage Dependence of Cell Viability}

The cell viability test was done according to the procedures supplied by the vendor, Sigma-Aldrich, which came with the product. Full strength distillates from distillation using $25 \mathrm{~g}$ of PV herb were used. The distillate from $25 \mathrm{~g}$ of herb was chosen because the distillate contained more diverse varieties of compounds including the rare ones; also, the abundances of others remained high.

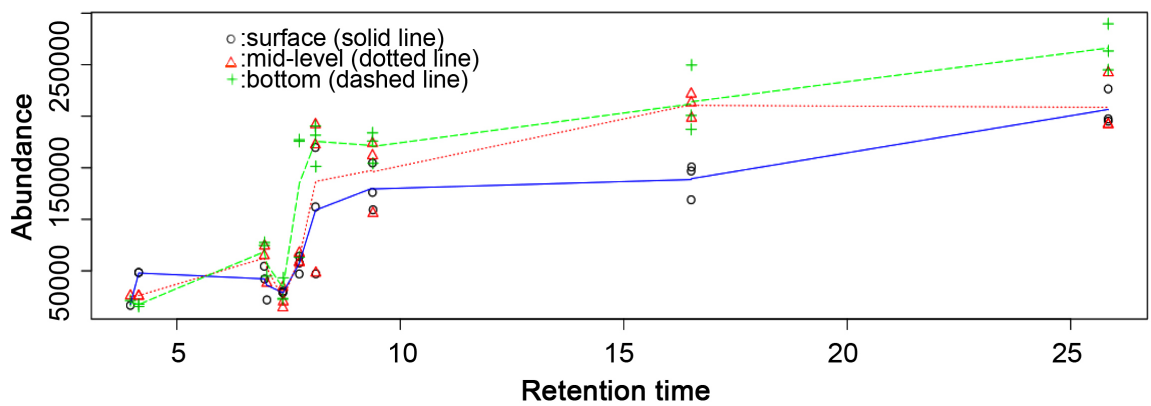

Figure 9. Abundance of various volatile compounds at three different depth levels. Note that compounds with very different abundances were not chosen to be included in this figure, as they will obscure the whole picture. (The specific identities of the individual compounds were not identified). 
They were calibrated to the standard concentration before they were used to treat the cells. With repeated dilution with de-ionized water, the distillates at different concentrations $(1,0.5,0.1,0.01 \mathrm{v} / \mathrm{v}$ of the full-strength distillate) were used to treat the cells, with 3 repeats each. After pre-incubation of the cells, the various concentrations of distillates were added to the culture medium in the wells. The plate was then further incubated for 48 hours before $10 \mu$ of CCK- 8 solution was added into the culture medium and incubated for another 3 hours. The absorbance at $450 \mathrm{~nm}$ using a microplate reader was then measured. Figure 10 shows the result. The optical density (od) shown on the y-axis is of arbitrary unit. The use of one-way ANOVA to analyse the statistical significance of the result was adopted. The null hypothesis was that PV solution of different concentrations made no differences in cytotoxicity, which means that the cytotoxicity was not dosage dependence. It gave an f-ratio value of 36.5 and the corresponding $\mathrm{p}$-value of less than 0.00001 . It can be concluded that the PV distillate was cytotoxic to the SCC154 cancer cells in a dosage dependent manner. However, since the differences in the cell viability are slight, the cytotoxicity of PV distillate on the cancer cell SCC154 is only mild.

\subsubsection{Cell Cytotoxicity of Different Distillate Portions}

Cell viability tests were also performed by treating the cancer cells using distillate portions obtained successively during the same distillation process using $25 \mathrm{~g}$ of herb. $10 \mu \mathrm{l}$ each of samples of distillate from different portion was added to the culture medium in the tissue culture plate to do the test. Figure 11 shows the results. One-way ANOVA was used to analyze the statistical significance. The null hypothesis was that there were no differences in cell cytotoxicity among the portions. This gave an f-ratio value of 5.825, and a p-value of 0.001329 , showing the differences in cell cytotoxicity among the portions were significant. It can be seen that the cell cytotoxicity was higher for portions obtained in the later part of

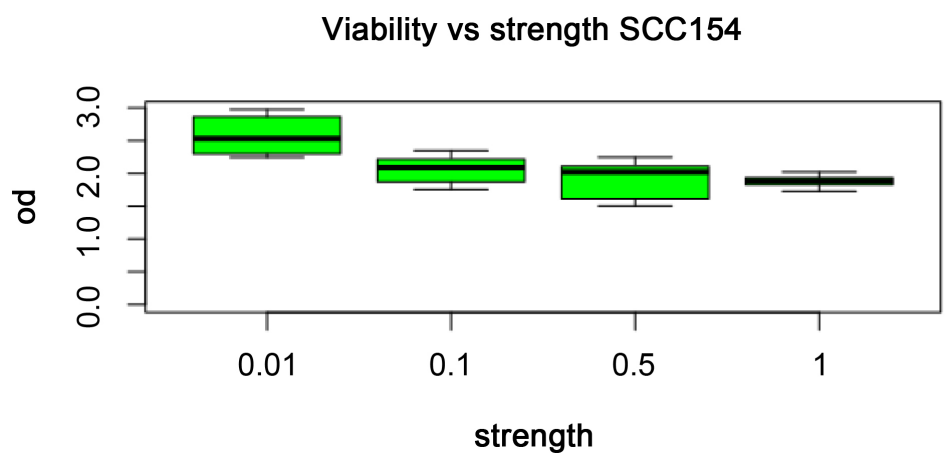

Figure 10. Cell viability plotted against the strength of the PV distillate. Different strengths were obtained by repeatedly diluting the full-strength solution with de-ionized water. Cell viability is indicated by the optical density of the absorbance. The full-strength distillate was from distillation using $25 \mathrm{~g}$ of herb. Portion 1 was used. The p-value for this set of data is, $\mathrm{p}<0.00001$, showing that the differences in cell viability due to the dilution of the PV distillate is significant. 


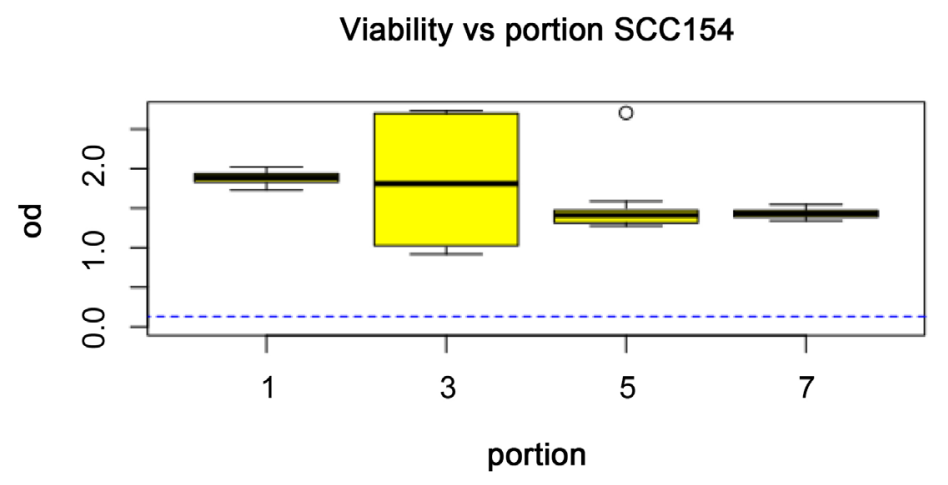

Figure 11. Cell viability of cells treated by the distillate obtained at different stages of the distillation process (as indicated by the successively obtained $50 \mathrm{ml}$ portions). $25 \mathrm{~g}$ of herb was used. The blue dotted line indicates the background level obtained by the negative control. Using ANOVA analysis, the p-value is 0.001329 , which is less than 0.05 , the usually chosen significant value. The optical density shown on the y-axis is of arbitrary unit.

the distillation process. As more volatile compounds deplete early in the distillation process, this observation hints that the anti-tumorous effect is due to compounds which are lower in their volatility, although their identities cannot be identified. However, since the differences in cell viability among the different portions are just slight, the differences of the cytotoxicity of the PV distillate at different points during the whole distillation process are just small, and so, they are approximately equally potent.

\subsubsection{Cell Cytotoxicity of Distillates from Distillation Using Different Weights of Herb}

Distillations were done using different weights of herb. Then, the distillates from such distillations were used to treat the cancer cells. The cell viability was again measured by the cell counting kit CCK-8. The result is shown in Figure 12 . Again, ANOVA was used to analyze the statistical significance of the result. The null hypothesis was that there were no differences in cytotoxicity for distillates prepared with different weights of herb. The statistical analysis gave an f-ratio value of 110.66 , and a p-value of less than 0.00001 . This shows that the differences of cell viability due to the use of different weights of herb are significant. By comparing Figure 12 with Figure 5 \& Figure 6, it can be seen that the cell viability results are consistent with the abundance curves in Figure 5 \& Figure 6. In Figure 12, the cell viability is least for the case when $15 \mathrm{~g}$ of herb used; while in Figure 5 \& Figure 6, the abundances of volatile compounds are highest also for the case when $15 \mathrm{~g}$ of herb used. The correspondence of these two different sets of data is obvious to provide another evidence that the PV distillate is cytotoxic to cancer cells SCC154 in a dosage dependent manner.

However, this conclusion was only drawn by the visual observation of the differences in the optical densities in Figure 12. Indeed, that the cell viability was least for the case when $15 \mathrm{~g}$ of herb used was confirmed by comparing the 
Viability vs weight SCC154

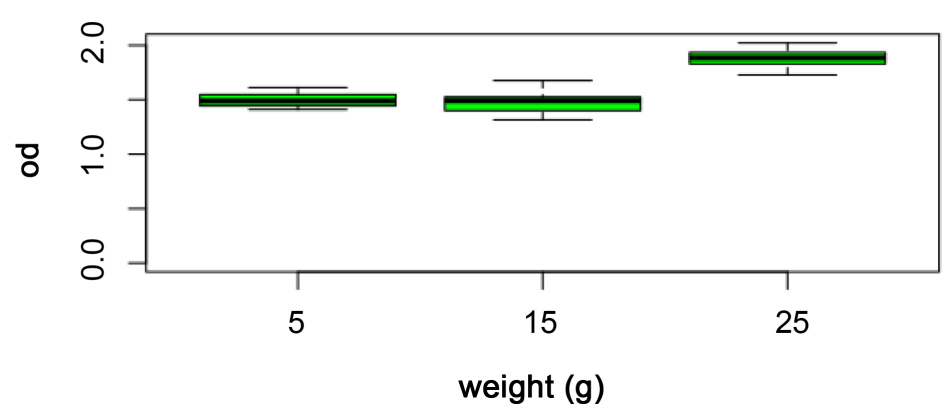

Figure 12. Cell viability of cells treated by the distillates from distillations using different amounts (weights) of herb. Portion 1 in each distillation was used. ANOVA analysis gave a p-value of less than 0.00001 . The optical density shown on the $y$-axis is of arbitrary unit.

sample means of the two groups: Optical densities were 1.5005 for the $5 \mathrm{~g}$ herb group, and 1.479 for the $15 \mathrm{~g}$ group. Further ANOVA analysis using these two groups alone gave a p-value of 0.47995 only, showing that the difference in cell viability between these two groups ( $5 \mathrm{~g}$ and $15 \mathrm{~g}$ herb used) is not statistically significant, however.

\section{Discussion}

The investigation focused on the characterization of the steam distillation process itself and the anti-tumorous effect of the volatile compounds as a whole in PV spica.

To fulfil these aims, the process of using steam distillation to extract the volatile compounds in PV was investigated to characterise the process. The results showed multiple volatile compounds were extracted at an approximately even abundance and rate over time (for as long as the distillation processes were allowed to proceed; see Figure 5 \& Figure 6); that is, the rate of extraction was approximately constant, even when the distillation went on for nearly the whole day. A limitation noted was the nature of the distillation apparatus setup (discussed further below) - varying amounts of raw PV did not vary the quantity of VOCs extracted, even when using a small quantity of PV.

The experimental setup of steam distillation with the PV herb mass located in the path of steam passage presented an obstacle for the efficient extraction of volatile compounds in hindsight. That is, even when more quantity of herb was used, even less volatile compounds could be extracted than with the initial $15 \mathrm{~g}$ of herbs. An explanation is that these VOCs in steam re-condensed and dropped back to the liquid being steamed and were unable to passage through the apparatus for collection.

Different arrangements in apparatus setup need to be investigated for steam distillation to overcome these limitations.

Other than the many volatile compounds which came out consistently even after the distillation process proceeded over time, the experiments showed that 
compounds such as benzaldehyde began to deplete over time, while some rarer compounds, such as eucalyptol, anisole, caryophyllene oxide, furfural, only appeared briefly at the beginning of the steam distillation VOC extraction process.

With respect to the PV therapeutic effects, only looking at the anti-tumorous effect of the PV distillate using the tetrazolium-based Cell Counting Kit- 8 was used as a proof of concept. It was found that PV distillate was cytotoxic to cancer cell SSC154 in a dosage dependent manner. Interestingly, distillate samples obtained later in the distillation process were more anti-tumorous than those obtained earlier. However, as seen from the experimental results, the cytotoxic effects can only be mild at best, and the differences among the time dependent extracted samples are only slight. Further work is required to identify which, if any, of the later occurring VOCs (or their absence from the earlier samples) might account for these differences observed.

In the GC-MS analysis, the VOCs were identified by comparing the mass spectra obtained with the mass spectrum library of the National Institute of Standards and Technology (NIST08). The accuracy of such identification method is limited as there is only high certainty (as reflected by the resemblance probability) if the compounds are abundant. However, the abundances of the volatile compounds in the herb are inherently low. The fact that the yield of volatile compounds in PV is small was also previously noted [5]. Thus, the identification of the VOCs in PV was accepted in this study using a less stringent criteria: That is, when the resemblance probability was high (identified as 50\%) in some of the corresponding chromatograms, groups of associated peaks in the neighborhood showed patterns to indicate the similarity of compounds, and the same peak with the same retention time appeared on different chromatograms. Next, the identities of the VOCs were confirmed by comparing the chromatograms with those of standard compounds available from the known chemical samples available in the laboratory.

While it was expected that the abundances of the compounds extracted would diminish as the distillation proceeded, this however was not observed. To clarify, a long distillation was done with $5 \mathrm{~g}$ of herb for 19 portions, which was equivalent to about 1 liter of distillate was collected which took about 15 hours. As shown in Figure 5(b) \& Figure 5(c), the abundances did not diminish substantially, even after such a long process of distillation. This, perhaps, reflected the abundance of compounds present.

The aim of using various quantities of PV herb $(2 \mathrm{~g}, 5 \mathrm{~g}, 15 \mathrm{~g}, 25 \mathrm{~g}, 35 \mathrm{~g}$, to 45 g) in this study was to see how the amounts of volatile compounds extracted depend on the amount of herb used. It was expected that when more herb was used, larger quantities of compounds should be extracted. This was seen when herb quantities were under $15 \mathrm{~g}$, increasing from $2 \mathrm{~g}, 5 \mathrm{~g}$ to $15 \mathrm{~g}$ (refer to Figure 5 \& Figure 6). However, when the amounts of herb used were increased further to $25 \mathrm{~g}$ and $35 \mathrm{~g}$, the abundances of volatile compounds were reduced. The curves in Figure 5 \& Figure 6 are similar, showing that they are closely bundled 
together. This means that the amounts of volatile compounds extracted were not sensitive to the amounts of herb used. However, this may indicate that the capacity limitations of the apparatus arrangement used to extract volatile compounds was saturated. That is (with reference to Figure 2), when steam passes through the herb, it will gradually be saturated by the volatile compounds from the herb. If the herb mass (quantity) is small, then saturation cannot be reached and thus the amount of volatile compounds extracted increases with the amount of herb used. Conversely, when larger herb quantities are used, saturation results which limits the amounts of volatile compounds extracted.

The loss (evaporation) of volatile compounds poses a problem in the research design using herbs with VOCs. As volatile compounds lose quickly with time, it is difficult to produce repeatable results, due to time dependent losses and storage environment of the PV herb. The strategy which was adopted in this study was to seal a fixed amount of PV herb samples (of $15 \mathrm{~g}$ each) from the larger package from the herb supplier, in plastic bags by heat sealing to minimize volatile compound loss and storing them in a closed dark cabinet. The sample seals were broken only when the experimental work was ready to proceed. Another strategy was to do the experiments, including the preparation of GC-MS solutions, the GC-MS analysis runs and the biological tests consecutively as soon as possible when the distillation is in progress, and as each individual distillate 50 $\mathrm{ml}$ sample was collected.

One uncontrolled concern was the source of the original PV herb. Because of the availability of the herb only through Chinese medicine herbal suppliers in Australia, the study was therefore limited to the use of dry herb (and as also used by registered Chinese medicine herbal practitioners). For this study, the herb was obtained from a local supplier who sources the herbs from China. Consequently, it was unknown from which locations the herb was sourced in China and the associated growing conditions (such as altitude and soil types), the age of the herb since harvest (and hence changes in volatile compounds), and effects of processing. In China, often different sourced batches of the herbs are mixed together for processing before sale to health practitioners. Consequently, the importer of the herbs could not provide details of the geographic origins, nor how the herbs from different regions were mixed. This is an important consideration. For example, a study by Yang et al. [33] compared the VOCs contents of PV from five Chinese provinces (Jiangsu, Fujian, Guangxi, Hunan and Zhejiang), reporting that PV samples from these different geographic locations showed different compound compositions. Thus, a future line of inquiry is to get a better understanding on the compound variations from the source plants in-situ and from a single origin.

In this work, the concentrations of volatile compounds in the aqueous distillate at different depths within the liquid were studied. Generally, heavier compounds sink to the bottom of the collection tube and lighter compounds float to the surface. This phenomenon can be used to fractionally concentrate the vola- 
tile compounds if particular compounds are the target of extraction.

The results showed wide variations of the data for the same experimental conditions, as shown by the triplicate sets of data. Variations can be minimised by mixing the distillate sample well before GC-MS analyses. This can be observed from the figures of results as shown above, after this small technique was adopted (Figure 5 \& Figure 6).

Another approach to minimize such variations is through increasing the number of data collection runs and eliminating the effect of wide variations by statistical means. However, the method of steam distillation as an extraction method has been reported previously as inherently time-consuming and inefficient [33], limiting how many data collection runs can be undertaken. Collection of a large database is thus difficult. An automated procedure may be a possible way to circumvent such difficulty.

For the anti-tumorous effect of the VOCs, a question which can be asked is whether the anti-tumorous effectiveness changes with successive portions taken during the distillation process (as used in this study). By treating the cancer cells with distillate samples from different portions, it was shown that the cytotoxicity, indeed, increases with the distillate samples taken later in the process, compared to earlier samples of the same distillation run. It is thus reasonable to speculate that those rarer compounds of higher volatility, such as eucalyptol, caryophyllene oxide, which occurred early in the distillation process and dissipated quickly and disappeared from later distillate portions, are not responsible for the cytotoxicity. What are responsible for the cytotoxicity of the PV volatile compounds should be the compounds with lower volatility (so that they persisted to appear in later portions also), although their identities have not been identified. Anyway, the differences in cytotoxicity among the different distillate portions are only slight (Figure 11). This implies a more relaxed requirement in the design of the medicine manufacture process using PV VOCs: The time to collect the VOCs during a distillation run is not critical, as the effectiveness of the VOCs collected does not differ much.

Finally, while steam distillation was investigated in this study, other extraction methods are available. These include hydro distillation, or might extend to other more efficient extraction methods, such as head-space solid-phase microextraction [42], or supercritical fluid extraction [43].

\section{Conclusions}

One of the aims of this study is to characterize the steam distillation process in the extraction of VOCs in PV. It was found that most VOCs came out evenly throughout the whole distillation process, except the rarer compounds. Additionally observed was that there was an optimal amount of herb used, with respect to the apparatus arrangement, provided the largest amounts of VOCs. This indicates that the steam distillation process undertaken has limited efficiency to extract VOCs from the PV herb, depending on the experimental setup. This 
study provides much insight in the design of a drug manufacturing process, which applies steam distillation to extract PVVOCs.

As for the anti-tumorous effect of the VOCs of PV, it was found that the VOCs are cytotoxic to the cancer cells from the SCC154 cell line in a dosage dependent manner. It was also found that the cytotoxicity of the VOCs extracted from PV was about the same, no matter when the VOCs were taken during the distillation process. This is a convenience to the design of the medicine manufacturing process.

\section{Conflicts of Interest}

The authors declare no conflicts of interest regarding the publication of this paper.

\section{References}

[1] Amjad, M.S., Qaeem, M.F., Ahmad, I., Khan, S.U., Chaudhari, S.K., Zahid Malik, N., Shaheen, H. and Khan, A.M. (2017) Descriptive Study of Plant Resources in the Context of the Ethnomedicinal Relevance of Indigenous Flora: A Case Study from Toli Peer National Park, Azad Jammu and Kashmir, Pakistan. PLoS ONE, 12, e0171896. https://doi.org/10.1371/journal.pone.0171896

[2] Rahman, I.U., Ijaz, F., Iqbal, Z., Afzal, A., Ali, N., Afzal, M., Khan, M.A., Muhammad, S., Qadir, G. and Asif, M. (2016) A Novel Survey of the Ethno Medicinal Knowledge of Dental Problems in Manoor Valley (Northern Himalaya), Pakistan. Journal of Ethnopharmacology, 194, 877-894.

https://doi.org/10.1016/j.jep.2016.10.068

[3] Siew, Y.Y., Zareisedehizadeh, S., Seetoh, W.G., Neo, S.Y., Tan, C.H. and Koh, H.L. (2014) Ethnobotanical Survey of Usage of Fresh Medicinal Plants in Singapore. Journal of Ethnopharmacology, 155, 1450-1466. https://doi.org/10.1016/j.jep.2014.07.024

[4] Wurtele, E.S., Chappell, J., Jones, A.D., Celiz, M.D., Ransom, N., Hur, M., Rizshsky, L., Crispin, M., Dixon, P., Liu, J.M., Po, W. and Nikolau, B.J. (2012) Medicinal Plants: A Public Resource for Metabolomics and Hypothesis Development. Metabolites, 2, 1031-1059. https://doi.org/10.3390/metabo2041031

[5] Golembiovska, O., Tsurkan, A. and Vynogradov, B. (2014) Components of Prunella vulgaris L. Grown in Ukraine. Journal of Pharmacognosy and Phytochemistry, 2, 140-146.

[6] Psotova, J., Kolar, M., Sousek, J., Svagera, Z., Vicar, J. and Ulrichova, J. (2003) Biological Activities of Prunella vulgaris Extract. Phytotherapy Research, 17, 1082-1087. https://doi.org/10.1002/ptr.1324

[7] Chen, J. K. and Chen, T. T. (2009) Chinese Herbal Formulas and Applications. Art of Medicine Press, CA.

[8] Scheid, V., Bensky, D. and Barolet, R. (2009) Chinese Herbal Medicine-Formulas and Strategies. Eastland Press, Washington DC.

[9] Qi, J., Hu, Z.F., Liu, Z.J. and Yu, B.Y. (2009) Triterpenes from Prunella vulgaris. China Journal of Natural Medicine, 7, 421-424. https://doi.org/10.1002/ptr.1324

[10] Cho, W.C.S. and Chen, H.Y. (2009) Transcatheter Arterial Chemoembolization Combined with or without Chinese Herbal Therapy for Hepatocellular Carcinoma: Meta-Analysis. Expert Opinion on Investigational Drugs, 18, 617-635. 
https://doi.org/10.1517/13543780902855308

[11] Zhang, M.Z. and Wang, X.Q. (2009) Effects of Extract from Prunella vulgaris Combined with Chemotherapeutic Agents on Proliferation of Lymphoma Cells. Tumor, 29, 961-964. [In Chinese]

[12] Feng, L., Jia, X.B., Zhu, M.M., Chen, Y. and Shi, F. (2010) Antioxidant Activities of Total Phenols of Prunella vulgaris L. in Vitro and in Tumor-Bearing Mice. Molecules, 15, 9145-9156. https://doi.org/10.3390/molecules15129145

[13] Oh, C., Price, J., Brindley, M.A., Widrlechner, M.P., Qu, L., McCoy, J.A., Murphy, P., Hauck, C. and Maury, W. (2011) Inhibition of HIV-1 Infection by Aqueous Extracts of Prunella vulgaris L. Virology Journal, 8, Article No. 188. https://doi.org/10.1186/1743-422X-8-188

[14] Chiu, L.C.M., Zhu, W. and Ooi, V.E.C. (2004) A Polysaccharide Fraction from Medicinal Herb Prunella vulgaris Downregulates the Expression of Herpes Simplex Virus Antigen in Vero Cells. Journal of Ethnopharmacology, 93, 63-68. https://doi.org/10.1016/j.jep.2004.03.024

[15] Yamasaki, K., Nakano, M., Kawahata, T., Mori, H., Otake, T., Ueba, N., Oishi, I., Inami, R., Yamane, M., Nakamura, M., Murata, H. and Nakanishi, T. (1998) Anti-HIV-1 Activity of Herbs in Labiatae. Biological and Pharmaceutical Bulletin, 21, 829-833. https://doi.org/10.1248/bpb.21.829

[16] Jun, M.S., Kim, H.S., Park, E.J., Lee, J.H., Lee, K.R., Kim, Y.S. and Chang, K.C. (2012) Ethanol Extract of Prunella vulgaris var. Lilacina Inhibits HMGB1 Release by Induction of Heme Oxygenase-1 in LPS-Activated RAW 264.7 Cells and CLP-Induced Septic Mice. Phytotherapy Research, 26, 605-612. https://doi.org/10.1002/ptr.3613

[17] Lee, I.K., Kim, D.H., Lee, S.Y., Kim, K.R., Choi, S.U., Hong, J.K., Lee, J.H., Park, Y.H. and Lee, K.R. (2008) Triterpenoic Acids of Prunella vulgaris var. Lilacina and Their Cytotoxic Activities in Vitro. Archives of Pharmacal Research, 31, 1578-1583. https://doi.org/10.1007/s12272-001-2154-6

[18] Lee, J.B., Kang, T.B., Choi, S.H., Lee, U., Kim, A.J., Jeong, C.J. and Lee, H.C. (2009) Effect of Prunella vulgaris Labiatae Extract on Innate Immune Cells and Anti-Metastatic Effect in Mice. Food Science Biotechnology, 18, 218-222.

[19] Kojima, H. and Ogura, H. (1986) Triterpenoids from Prunella vulgaris. Phytochemistry, 25, 729-733. https://doi.org/10.1016/0031-9422(86)88033-5

[20] Du, D., Cheng, Z. and Chen, D. (2012) A New Unusual delta11(12)-oleane Triterpene and Anti-Complementary Triterpenes from Prunella vulgaris Spikes. Natural Product Communications, 7, 501-505.

[21] Choia, H.G., Kim, T.H., Kim, S.H. and Kim, J.A. (2016) Anti-Allergic Inflammatory Triterpenoids Isolated from the Spikes of Prunella vulgaris. Natural Product Communications, 11, 31-32. https://doi.org/10.1177\%2F1934578X1601100111

[22] Yang, J., Hu, Y.J., Yu, B.Y. and Qi, J. (2016) Integrating Qualitative and Quantitative Characterization of Prunellae Spica by HPLC-QTOF/MS and HPLC-ELSD. Chinese Journal of Natural Medicines, 14, 391-400.

[23] Gu, X.J., Li, Y.B., Mu, J. and Zhang, Y. (2011) A New Phenolic Glycoside from Prunella vulgaris. Acta Pharmaceutica Sinica, 46, 561-563.

[24] Sahin, S., Demir, C. and Malyer, H. (2011) Determination of Phenolic Compounds in Prunella L. by Liquid Chromatography-Diode Array Detection. Journal of Pharmaceutical and Biomedical Analysis, 55, 1227-1230. https://doi.org/10.1016/j.jpba.2011.03.016

[25] Xu, H.X., Lee, S.H., Lee, S.F., White, R.L. and Blay, J. (1999) Isolation and Characte- 
rization of an Anti-HSV Polysaccharide from Prunella vulgaris. Antiviral Research, 44, 43-54. https://doi.org/10.1016/S0166-3542(99)00053-4

[26] Feng, L., Jia, X.B., Shi, F. and Chen, Y. (2010) Identification of Two Polysaccharides from Prunella vulgaris L. and Evaluation on Their Anti-Lung Adenocarcinoma Activity. Molecules, 15, 5093-5103. https://doi.org/10.3390/molecules 15085093

[27] Li, C., You, L., Fu, X., Huang, Q., Yu, S. and Liu, R.H. (2015) Structural Characterization and Immunomodulatory Activity of a New Heteropolysaccharide from Prunella vulgaris. Food and Function, 6, 1557-1567. https://doi.org/10.1039/C4FO01039F

[28] Liao, L., Guo, Q.S., Liu, L. and Liu, M. (2008) Study of Flavonoids Distribution in Various Populations of Prunella vulgaris. China Journal of Chinese Materia Medica, 33, 651-653.

[29] Wang, Y., YIN, J., Guo, Q. and Xiao, Y. (2011) Dynamic Change of Active Component Content in Different Parts of Prunella vulgaris. China Journal of Chinese Materia Medica, 36, 741-745.

[30] Yang, Y.L., Shi, F., Jian, G.P., Ouyang, Y. and Zhao, H.X. (2013) Extraction of Volatile Oil from Artemisia argyi Leaves and Component Analysis. Journal of Anhui Agricultural Science, 41, 5267-5271.

[31] Tomaino, A., Cimino, F., Zimbalatti, V., Venuti, V., Sulfaro, V., De-Pasquale, A. and Saija, A. (2005) Influence of Heating on Antioxidant Activity and the Chemical Composition of Some Spice Essential Oils. Food Chemistry, 89, 549-554. https://doi.org/10.1016/j.foodchem.2004.03.011

[32] Morteza-Semnani, K., Saeedi, M. and Akbarzadeh, M. (2006) The Essential Oil Composition of Prunella vulgaris L. Journal of Essential Oil Bearing Plants, 9, 257-260. https://doi.org/10.1080/0972060X.2006.10643500

[33] Yang, Y., Nan, H., Wang, G., Yang, W. and Xu, J. (2013) Comparative Determination of the Volatile Components of Prunella vulgaris L. from Different Geographical Origins by Headspace Solid-Phase Microextraction and Gas Chromatography-Mass Spectrometry. Analytical Letters, 46, 2001-2016.

https://doi.org/10.1080/00032719.2013.782551

[34] Zhai, X., Xi, M.Q., Guo, Q.S., Han, H.H., Zhang, X., Yang, W., Zheng, R.B., Huang, X.D. and Zhu, H.R. (2014) Preliminary Analysis of Bitter Substances in Spica of Prunella vulgaris. China Journal of Chinese Materia Medica, 39, 423-426.

[35] Sun, C. (2017) A Review on Pharmacological Effects of Xiakucao. Clinical Journal of Chinese Medicine, 9, 146-148.

[36] Zhao, X.-M. (1765) Addendum to the Ben Cao Gang Mu [本草纲目拾遗]. Chinese Medicine Publications, Beijing.

[37] Chen, C.Z. (2016) Treatment Methods and Formulae in Chinese Medicine. People Health Publishing Co., Beijing.

[38] Gu, X.J., Qian, S.H., Li, Y.B., Li, P. and Duan, J.A. (2007) The Progress in the Research of the Chemical Composition and the Pharmacological Effects of Prunella vulgaris. Chinese Wild Plant Resources, 26, 5-7.

[39] Wang, H.B., Zhang, Z.Y. and Su, Z.W. (1994) The Constituents of the Essential Oil from Three Plants of Prunella vulgari. China Journal of Chinese Materia Medica, 29, 652-653.

[40] Cai, X., Peng, S., Shi, J.L. and Li, C.R. (2005) In Vitro Antibacterial Activities of 5 Traditional Chinese Medicines against Bacteria Strains Commonly Encountered in the Clinic. Herald of Medicine, 24, 470-472. 
[41] Chinese Pharmacopoeia Commission (2010) Pharmacopoeia of the People's Republic of China. Vol. 1, China Medical Science Press, Beijing, 359.

[42] Adam, M., Juklova, M., Bajer, T., Eisner, A. and Ventura, K. (2005) Comparison of Three Different Solid-Phase Microextraction Fibres for Analysis of Essential Oils in Yacon (Smallanthus sonchifolius) Leaves. Journal of Chromatography A, 1084, 2-6. https://doi.org/10.1016/j.chroma.2005.05.072

[43] Sarker, S.D., Latif, Z. and Gray, A.I. (2006) Natural Products Isolation. Humana Press, Totowa. 